\title{
INTERVENTIONS FOR INDIVIDUALS WITH YOUNG-ONSET DEMENTIA. A REVIEW OF THE LITERATURE
}

\author{
M. Aplaon1, P. Belchior1,2, I. Gélinas 1,3, N. Bier2,4, A. Aboujaoudé1,2
}

\begin{abstract}
Objective: The aim of this study was to conduct a scoping review of the literature to investigate non-pharmacological interventions for individuals with young-onset dementia and examine their success. Methods: A comprehensive review of the literature was conducted. The following databases were included in our search: Ovid Medline, PsycINFO, Pubmed, CINHAL. Studies were retained if they were 1) peer-reviewed; 2) published in English; 3) focused on non-pharmacological interventions; 4) designed for persons with dementia; and 5) diagnosis before the age of 65. Results: A total of 7 articles met our inclusion criteria and were included in the study. Of those, four consisted in social programs involving the community as part of the intervention, two focused on a cognitive based intervention and one focused on a cognitive behavioral intervention. The social interventions were successful in improving the mood and well-being of participants as well as providing respite for caregivers. The cognitive interventions were successful in improving perceived functional outcomes and affective symptoms of participants. Discussion: A discussion of each intervention including their benefits for individuals with young-onset dementia and their caregivers is presented.
\end{abstract}

Key words: Young-onset dementia, early-onset dementia, intervention.

\section{Introduction}

The number of individuals living with dementia has increased dramatically over the years. The burden of the disease is enormous for both the affected individuals and their family members. In addition, to date, the healthrelated costs of the disease amount to $\$ 33$ billion per year (1). While Alzheimer's disease (AD) and related dementias are often assumed to only affect older adults, approximately 1:1000 individuals suffer from youngonset dementia (YOD) (2).

In the literature, the terminologies of 'young-onset dementia' (YOD) and 'early-onset dementia' (EOD) are often used interchangeably. They refer to the condition when the symptoms manifest before the age of 65 . On the other hand, the symptoms appear after the age of 65 in 'late-onset dementia' (LOD). For the purpose of this scoping review, YOD and EOD will be treated as the same and YOD will be used throughout the text.

Although the prevalence of YOD is lower in comparison to that of LOD, it still affects a significant number of individuals. To date, studies have identified

1. McGill University, Montréal, Canada; 2. Institut universitaire de gériatrie de Montréal, Montréal, Canada; 3. Centre de recherche interdisciplinaire en réadaptation du Montréal métropolitain, Montréal, Canada; 4. Université de Montréal, Montréal, Canada

Corresponding Author: P. Belchior: 3654 Promenade Sir-William-Osler (Drummond), Montréal, Qc, Canada; e-mail: patricia.belchior@mcgill.ca; phone number: 5143984400 ext 00675 the most frequent causes or etiology of YOD as being $\mathrm{AD}$, vascular dementia, and frontotemporal dementia (FTD) $(4,5)$. In a previous study, AD accounted for $34 \%$ of the cases, vascular dementia 18\%, and FTD 12\% (5). Other reported characteristics of YOD include: changes in memory, attention, and personality, as well as executive dysfunction and behavioral disinhibition (3). Additionally, some have tried to compare the cognitive, behavioral, and psychological symptoms of YOD and LOD to better understand their differences $(6,7)$.

Taking into consideration that people with LOD are mostly retired, have no dependents, and are mortgagefree, while people with YOD are usually physically strong and healthy, generally working at the time of the diagnosis, supporting dependent children and/or aging parents, and have significant financial obligations and commitments (2), the impact and burden of YOD can be far greater and far more overwhelming for this population.

Given their younger age, individuals with YOD would require different types of support and services than those available for individuals with LOD. To date, no study has investigated current psychosocial programs including cognitive training developed precisely for individuals with YOD to address their specific needs. Thus, the purpose of this study was to conduct a scoping review of the literature to investigate different non- 
pharmacological interventions available for individuals with YOD and examine their success.

\section{Methodology}

\section{Search strategy}

A review of the literature was conducted with guidance from an experienced health sciences librarian. Articles retained met the following criteria: 1) peer-reviewed; 2) published in English; 3) focused on non-pharmacological interventions, specifically psychosocial interventions including cognitive training; 4) for persons with dementia, or other related diseases; 5) diagnosed before the age of 65 . Studies which comprised of a combination of pharmacological and non-pharmacological interventions were excluded. Four electronic databases were searched until March 2016 (CINAHL plus with full text, OvidMedline from 1996, PsychINFO from 2002, and Pubmed). Mesh terms and keywords were used for Pubmed, and subject headings and keywords were used for the other databases. The following search combination was used: Dementia (Mesh or subject heading) OR dement* (keyword) OR Alzheimer Disease (Mesh or subject heading) OR alzheimer* (keyword) AND early onset or young* onset (title) AND program* or club (keyword) OR Rehabilitation (Mesh or subject heading) OR rehabilit* (keyword) OR Resistance Training (Mesh or subject heading) OR training (keyword) OR intervention* (keyword) OR activit* (keyword) OR service* (keyword). The reference lists of eligible publications were also retrieved and reviewed.

\section{Results}

A total of 398 articles were retrieved from the literature search (120 from CINAHL, 86 from OvidMedline, 64 from PsychINFO, 128 from Pubmed). Among those, 172 articles were duplicates and were removed. In total, 226 articles title and abstract were screened and 10 potential articles were identified. Further review of the articles revealed that only 7 explicitly examined an intervention program for people diagnosed with YOD and met all the inclusion criteria (see figure 1). Of those, four consisted of social programs involving the community as part of the intervention, two were case reports focused on a cognitive training based intervention, and one was a case report focused on a cognitive behavioral intervention. The details of each program can be seen in table 1.

The psychosocial interventions included participation in a community sports program (10), a gardening program (11), or a workplace engagement program at a hardware store (12) and at the zoo (14). Three consisted in feasibility studies $(10,12,14)$. Most of the interventions had qualitative outcomes assessed through focus groups, informal or more structured interviews. Notably, they all reported common beneficial outcomes from participants and caregivers perspectives $(10,12,14)$ or only caregivers (11), including improved mood and well-being arising from socialization opportunities, a sense of belonging and normalcy, and feeling useful and valued through accomplishing meaningful tasks. Another common benefit was the provision of respite for caregivers (10, $11,12,14)$. Moreover, one study particularly finds benefits lasting beyond the program enrollment (14). Interestingly, one study found increased participants' well-being, assessed quantitatively through the pool activity level instrument, despite cognitive decline measured by mini-mental state examination at 6-months and 1-year follow-up (11).

\section{Figure 1}

Flow diagram of article inclusion (8)

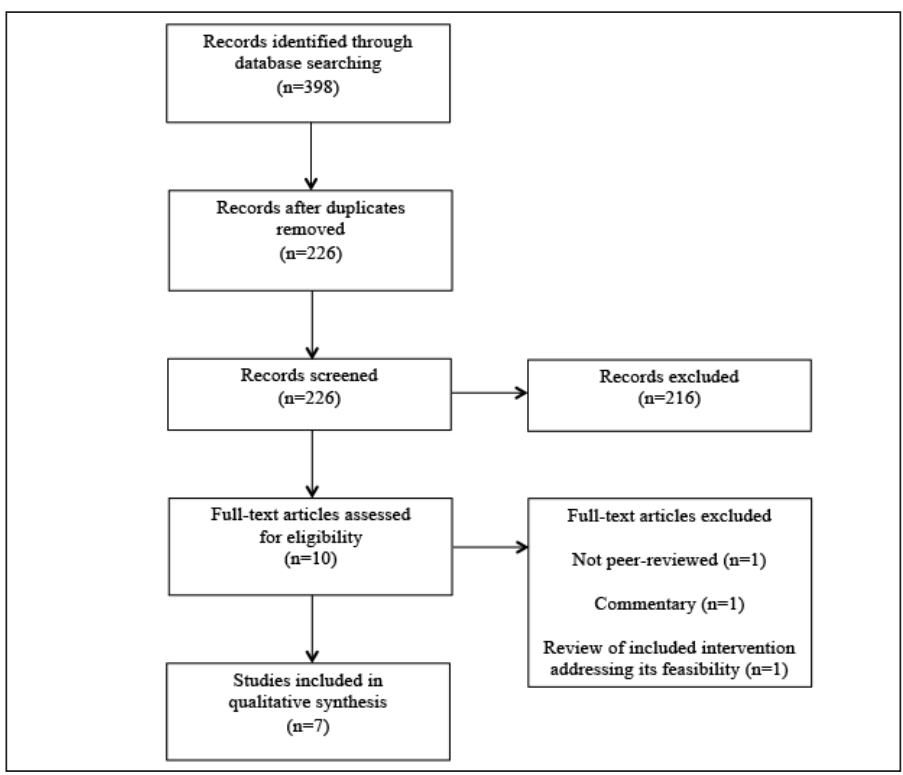

The cognitive interventions results are all founded on case-reports. The first intervention was tailored to an individual with cerebral autosomal dominant arteriopathy with subcortical infarcts and leukoencephalopathy and followed the Attention Process Training II manual complemented with strategy training activities (13). It demonstrated improved performance of processing speed and executive functions as well as perceived functional outcomes posttreatment (13). The second intervention, tailored specifically to the needs and complaints of an individual with posterior cortical atrophy (PCA), consisted of sessions of psychoeducation, cognitive exercises for visual/recognition training and compensatory strategies, supplemented with home activities including the repetition of taught strategies (15). While it demonstrated slight improvement on test results following initial treatment, this improvement was not maintained when the training was prolonged for an additional 12 months, although perceived improvement 


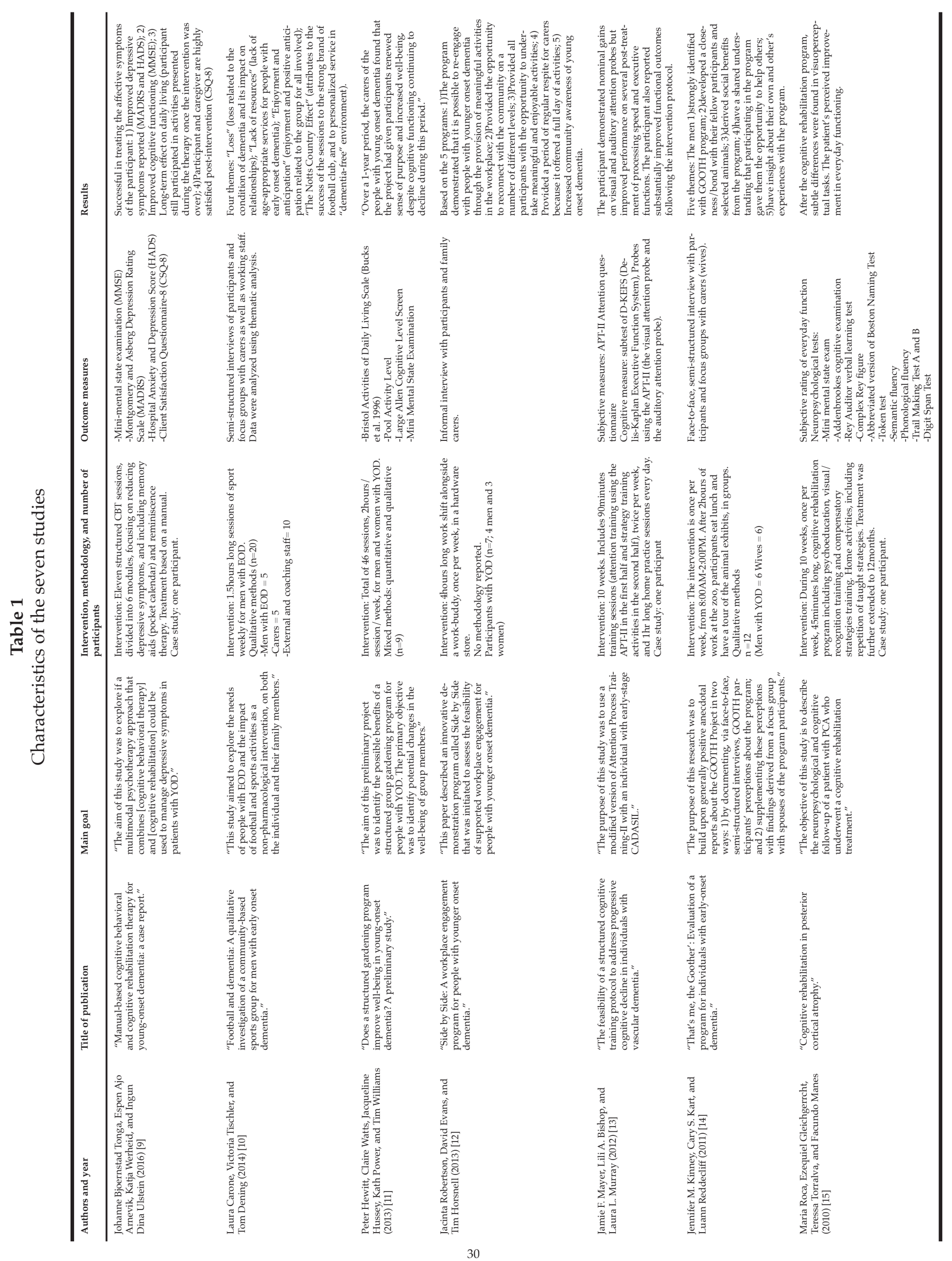


in everyday functioning was reported (15). The last intervention, for an individual with YOD, consisted in a structured manual-based cognitive training behavioral program divided into 11 sessions targeting reducing depressive symptoms (9). It demonstrated that making a list of pleasurable activities as well as usage of a pocket calendar, to help keep a record of meaningful activities completed, improved depression symptoms during the intervention. Moreover, long-lasting satisfaction was expressed by the participant and the caregiver, following the intervention, despite pocket calendar abandonment due to forgetfulness (9).

\section{Discussion}

At present, since there are fewer individuals diagnosed with YOD compared to LOD, people with YOD are forced to fit into a system of services designed and intended for people with LOD $(10,11)$. Caregivers report the lack of age-appropriate programs, or services, available which translates into feelings of frustration, neglect, and heightened isolation in individuals with YOD $(10,14)$ that could be minimized through meaningful and purposeful interventions.

This scoping review identified seven programs for individuals with YOD. Four consisted of psychosocial interventions and 3 of cognitive interventions. The cognitive training interventions were successful in improving perceived functional outcomes $(13,15)$ and affective symptoms (9). The structured social engagement interventions have proven to be worthwhile from participants and caregivers perspectives.

The psychosocial interventions were considered successful for the following reasons: 1) the natural environment engagement gave participants a feeling of normalcy, which people with YOD struggle to achieve after owing to the perceived stigma that the society places on a diagnosis of dementia $(10,14) ; 2$ ) by accomplishing concrete tasks they were able to feel useful, valued, learn new skills, and find a sense of purpose (10, 11, 12, 14); 3 ) they found a sense of belonging in their group which provided them with peer support and non-judgmental social interactions $(10,11,12,14)$.

The results of this scoping review should be taken with caution considering its limitations. First, only English articles were retrieved. Thus, it is possible that our search missed publications in a different language. In addition, because the terminology young-onset/early-onset dementia is relatively new, we might have missed articles that did not contain these terms. Moreover, the retained and analyzed studies all contained 1) a very small sample size; 2) more men than women; and 3) an individualistic approach (i.e. almost a one-on-one participant-tostaff ratio). Considering these, and as mentioned in every article, it is not possible to draw a generalizable conclusion. The fact that almost all programs had a centered approach specifically tailored to each participant further prevents the possibility to generalize. Finally, the exclusion of unpublished literature could induce publication bias.

\section{Conclusion}

Our research yielded seven intervention programs for individuals with YOD. Every intervention reported positive benefits for participants and their caregivers. Nonetheless, it is noteworthy that there are very few studies that explore, document, and assess the benefits of programs for individuals with YOD. Given the particularities of people suffering from YOD, there is a growing need to develop, study, review, and establish effective interventions that respond to the unique needs of this population.

Conflict of interest: None.

Ethics Standards: Not applicable.

\section{References}

1. A new way of looking at the impact of dementia in Canada. Alzheimer Society Canada. 2012. http://www.alzheimer.ca/ / media/Files/national/ Media-releases/asc_factsheet_new_data_09272012_en.pdf. Accessed 10 November 2015

2. Young onset dementia. Alzheimer Society Canada. 2015. http://www. alzheimer.ca/en/About-dementia/Dementias / young-onset-dementia. Accessed 10 November 2015

3. Kelley, BJ., Boeve, BF., Josephs, KA. Cognitive and noncognitive neurological features of young-onset dementia. Dementia and Geriatric Cognitive Disorders, 2009;27, 564-571.

4. McMurtray, A., Clark, D., Christine, D., and Mendez, M. Early-onset dementia: frequency and causes Compared to late-onset dementia. Dementia and Geriatric Cognitive Disorders, 2005;21, 59-64.

5. Harvey, R., Skelton-Robinson, M., \& Rossor, M. The prevalence and causes of dementia in people under the age of 65 years. Journal of Neurology, Neurosurgery \& Psychiatry, 2003;74(9), 1206-1209.

6. Licht, E., McMurtray, A., Saul, R., and Mendez, M. Cognitive differences between early- and late-onset alzheimer's disease. American Journal of Alzheimer's Disease \& Other Dementias, 2007;22, 218-222.

7. Toyota, Y., Ikeda, M., Shinagawa, S., Matsumoto,T., Matsumoto, N., Hokoishi, K., Fukuhari, R., Ishikawa, T., Mori, T., Adachi, H., Komori, K., and Tanabe, H. Comparison of behavioral and psychological symptoms in earlyonset and late-onset Alzheimer's Disease. International Journal of Geriatric Psychiatry, 2007;22, 896-901.

8. Moher, D., Liberati, A., Tetzlaff, J., Altman, D. G. and The PRISMA Group. Preferred Reporting Items for Systematic Reviews and Meta-Analyses: The PRISMA Statement. PLoS Medicine. 2009;6(7).

9. Tonga, JB., Arnevik, EA., Werheid, K., Ulstein, ID. 'Manual-based cognitive behavioral and cognitive rehabilitation therapy for young-onset dementia: a case report.' Int Psychogeriatr, 2015;28(3), 519-22.

10. Carone, L., Tischler, V., and Dening, T. Football and dementia: A qualitative investigation of a community based sports group for men with early onset dementia. Dementia, 2014;0(0) 1-19.

11. Hewitt, P., Watts, C., Hussey, J., Power K., and Williams, T. Does a structured gardening programme improve well-being in young-onset dementia? A preliminary study. British Journal of Occupational Therapy, 2013;76(8), 355361.

12. Robertson, J., Evans, D., and Horsnell, T. Side by Side: A workplace engagement program for people with younger onset dementia. Dementia, 2013;12(5), 666-674.

13. Mayer, J., Bishop, L., and Murray, L. The feasibility of a structured cognitive training protocol to address progressive cognitive decline in individuals with vascular dementia. American Journal of Speech-Language Pathology, 2012;21, 167-179.

14. Kinney, J., Kart, C., and Reddecliff, L. 'That's me, the Goother': Evaluation of a program for individuals with early-onset dementia. Dementia, 2011;10(3), 361-377.

15. Roca, M., Gleichgerrcht, E., Torralva, T., and Manes, F. Cognitive rehabilitation in posterior cortical atrophy. Psychology Press, 2010;20 (4), 528-540. 